\section{SOME COMMON CAUSES OF STERILITY AND THEIR TREATMENT.}

\author{
By ARTHUR GRAY, \\ F.R.C.S. \\ Gynacological Surgeon, Hampstead General Hospital \\ and Miller General Hospital.
}

THE investigation and treatment of many cases of sterility are simple, in others, the cause though present, is likely to be overlooked. This paper is concerned with the commonly overlooked causes of sterility and with the method of investigation and treatment of any particular case in which the cause is not reasonably obvious, as within the last few years, considerable advance has been made on this side of the subject, and it is well to have a cut-and-dried method of procedure in investigating these cases.

\section{DEFINITION OF STERILITY.}

Duncan's definition is the best : "That it is generally considered to imply the condition of a woman who, under ordinary circumstances for reproduction, does not bring forth a living child."

Sterility may be Artificial or Natural.In the latter case, it may be absolute or relative.

In absolute sterility, conception has never occurred and cannot occur without treatment.

In relative sterility, sometimes called onechild sterility, the foetus is either cast off before viability, or one child is born and then no more. It is in this particular group that great advance has been made with regard to treatment.

It is obvious that the time which elapses between the date of marriage and the date of conception is a very important one for the woman as a test of her fertility, because if it goes much beyond the average she must be considered relatively sterile.
When can a Marriage be Considered Sterile and Therefore Treatment JUSTIFIED?

Whether the woman will be sterile or not, is practically decided within three years of the date of her marriage, since as a matter of fact only 7 per cent. bear children after that time.

Kish gives the following figures: In 536 fruitful marriages, first delivery occurred within ten months in 156 ; within eleven to fifteen months in I99; within sixteen to twenty-four months in II 5 ; in two to three years in 6o; more than three years in 26 .

Thus, in 35.5 the first delivery occurred within fifteen months of marriage.

Women marrying after 25 years of age are longer before conception takes place than those between 20 and 25 years.

\section{FACtors Influencing Fertility.}

The Influence of Age.-Age is of more importance in the woman than in the man. Extreme youthfulness and too advanced age impair fertility.

In the case of roo marriages in which the wife's age was 18 to 20 , the average offspring was 40 (this is the maximum): At 25 the average is 32 ; at 30 the average is 24 ; at 35 the average is 17 ; at 40 the average is 10 ; at 45 the average is 7 .

It is obvious that in ascertaining the fertility of the woman, the age of the husband requires consideration as it exercises an important influence on the wife's fertility. To put it as tersely as possible: "The maximum fertility is shown by a woman of $\mathbf{I} 8$ to 20 married to a man of 25 ; by a woman of 29 married to a man of 29 ; by a woman of 30 years and upwards married to a man younger than herself. It follows from this that those marriages will be most fruitful in which husband and wife are of the age most favourable to fertility, thus, the ideal marriage is one in which the wife is 20 years and the husband 24 to 29 years of age.

The Marriage of Near Kin.-It is estimated 
that roughly 32 per cent. of these marriages are sterile. The average interval between successive births of children is of importance in estimating the presence of relative sterility. The average interval is about twenty months, thus it follows: If a woman conceives at the usual time after marriage and bears a second child about twenty months after the first, she is not relatively sterile, but if three years elapse, other things being equal, she must be included in this category. In this connection one has to bear in mind that just as members of large families tend to have large families themselves, so some families tend to die out.

With the above facts in mind, one can turn to what I consider to be some of the causes of sterility which are commonly overlooked.

Many causes are obvious on examınation ; in fact, the woman or her husband may' tell you the reason before examination. It is likely that any condition causing dyspareunia, or vaginismus, any deformity or maldevelopment, inflammatory condition, or obstruction to coitus, will or at least may result in failure to conceive. It is not with these causes I wish to deal but rather with those more obscure.

The first of these involves an examination of the male partner, since in one in five cases the husband is the cause.

It is only of recent years that this fact has been recognized, formerly it was always the woman considered to be at fault, but in future no woman need fear she will be classed as sterile, until her husband's inadequacy has been excluded, and, furthermore, no woman in whom there is no obvious cause should be advised to undergo an operation for the cure of sterility unless her husband is known to be fertile.

The Causes of Sterility in the Male.

Conditions Preventing or Interfering with Coitus.-Such are congenital malformations or a tight stricture, and conditions of nervous origin, such as premature emission.
It is in this group of causes that examination of semen in a condom specimen is fallacious.

There are several reasons why the mere finding of live spermatozoa in such a specimen is not sufficient evidence to prove the the male element is satisfactory.

Hypospadias and epispadias may be present, and as a consequence live spermatozoa may be recovered from the condom, when in the normal act they do not reach the cervix. So, too, if emission is premature, semen will be found in the condom.

In cases of stricture of the urethra, live spermatozoa may be found in semen that has dribbled through the stricture and been retained in the condom after withdrawal of the penis.

The proper method of investigating the -male will be discussed under the heading of treatment.

Conditions Affecting the Development of the Sperm or its Fertilizing Power.-We know that conception does not always depend on the motility of the spermatozoa alone, but upon the site in which they are deposited. Thus spermatozoa deposited in the vagina are very soon killed by the vaginal acidity, the alkaline reaction of the prostate fluid only neutralizing the acidity for a very short time. On the other hand, spermatozoa deposited on the cervix, the secretion of which is alkaline, are able to survive for a considerable period.

On examination of the seminal fluid, aspermia, that is absence of the spermatozoa, or oligospermia (i.e., few sperm), may be found. Common causes of these conditions are old age, debility, and prostatic atrophy, but the two most important are gonorrheca and sexual excess, gonorrhoa affecting the vas, epididymus and testis. Probably 75 per cent. of men attacked with gonorrhœa epididymitis become sterile. In this connection it must be remembered that spermatozoa may be present after an attack and still have lost their fertilizing power. Thus it is well known if the sperm of a frog be frozen and then allowed to thaw, the spermatozoa 
recover their motility but become incapable of fertilizing an ovum. Syphilis is a much less common cause of sterility in man.

Sexual excess is another important cause in the man. As a rule it takes three days for more spermatozoa to form capable of fertilizing an ovum after coitus, the immature forms in the semen during this period being useless for this purpose. Sanger found, on inspection of the male, in I Io sterile marriages that: 53.6 per cent. showed normal sperm ; I I.8 per cent. showed oligospermia ; $33^{\circ} 6$ per cent. showed aspermia.

It is very important to remember that although a man be examined many times and show absence of spermatozoa sometimes, years later, a few spermatozoa appear and cause fertilization. This is probably an explanation of sterile unions, the wife being healthy, in which conception takes place after years of married life. Thus it is important that the doctor should not definitely commit himself if he finds aspermia in the male.

Under what conditions can the man be e.xcluded? - It can be stated quite definitely that if live and active spermatozoa are found to be deposited on the cervix after coitus, then the husband is normal and the fault is very unlikely to be his.

Before leaving the male as the cause I would like to mention two other important factors, namely, general debility-often a long holiday will suffice to cure. The other is obesity. This in some way seems to affect the male fertilizing power. It may be due to atrophy of the testicles, but there is no doubt that as the ordinary rate of barren to fruitful marriages is I to ro, in eases in which either the wife or both the wife and husband are obese, the sterility ratio is doubled (I to 5$)$.

\section{The Causes of Sterility in THE FEMALE.}

The first one I will mention is uterine displacement.

(I) Acute Anteflexion. We all know that conception can and does take place with this condition present. In facl, to a greater or lesser extent, it is present in 20 per cent. of women, but the percentage is much higher in those seeking relief for sterility. The cause of the sterility in some of the cases lies in the presence of small polypi or nodular excrescences lying in the cervical canal which by their secretions inhibit the action of the spermatozoa.

(2) Retronersion and retroflexion are very common conditions. They often cause no symptoms and are only discovered on examination. They may produce sterility in one of several ways. The position of the os is unfavourable, an erosion is often present, especially on the posterior lip of the cervix and its secretion may be inimicable to the sperm; lastly the mere backward displacement of the uterus may, by its pressure, obstruct the tubes. This has been actually demonstrated by tubal inflation via the cervix, with the abdomen open, so that the tubes can be seen at the time of inflation.

\section{Tubal and Orarian Causes.}

There is no doubt that salpingitis is the commonest cause of sterility in women, and gonorrhoea is the commonest cause of the salpingitis (probably 70 per cent.), thus gonorrhoa is the commonest cause of absolute and relative sterility in women (probably 50 per cent. of all cases). Of course the other causes of salpingitis play a part, such as childbirth infection, and other causes of peritonitis, leading to adhesions and tubal obstruction, such as appendicitis, ruptured gastric ulcer and tuberculous peritonitis.

It is not essential that there should be absolute tubal obstruction, for should the spermatozoa succeed in escaping the uterine infection, they still may be unable to survive the discharge in the tube. Thus 50 per cent. of women who are curetted for sterility, will remain sterile if nothing more is done.

The types of tubal obstruction commonly found are : occlusion of the ostium by indrawing of the fimbria and adhesions, 
without any distension of the tubal lumen. This type is favourable for treatment whilst closure of the ostium with distension of the tube usually means that the uterine end of the tube is also closed, when the chances of pregnancy are very poor after operation.

The commonest overlooked ovarian condition causing sterility is thickening of the tunica albuginea, the result of inflammation. This causes obstruction to ovulation and a persistent corpus luteum will suppress further ovulation. We know that it requires only a very small amount of ovarian substance to allow the continuance of menstruation and therefore the possibility of conception and there is no doubt that ovarian sterility is much more frequently caused by some inflammatory condition of the surface of the ovary, possibly by extension from the tube or appendix, than by actual affection of the ovarian tissue itself, as pregnancy does occur in the presence of ovarian tumours when both ovaries have been affected by the growth, and have grown to considerable size.

Conditions Preventing Development of the Fertilized Ovum.-These may be mechanical, such as severe lacerations of the cervix, leading to loss of sphincter control and repeated abortions. Unsuitable soil. By this I mean an unhealthy cervix (endocervicitis, cervical erosion) or an unhealthy endometrium, either true endometritis, or polypoid hyperplasia. The unhealthy discharges from the cervix are inimicable to the spermatozoa so that conception is prevented. In the case of the endometrium, it may be that this interferes with the syncytial development, or some obscure biochemical change brought about by the mucous membrane acts upon the ovum, indeed the actual organisms in the endometrium may attack the ovum. A very common history in this type of case is as follows : Every now and again the period is a few days late; when it comes on it is very free and lasts a day or two longer than is usual. These are really repeated early abortions, and if a curettage is performed in this type of case, normal pregnancy often follows. These patients complain of the usual symptoms of so-called endometritis.

Alteration in the Vaginal Secretions.Normally, the vaginal secretion is acid and inimicable to the spermatozoa. This is due to the presence of Doderlein's bacillus, which produces lactic acid. The normal acidity is equivalent to 0.4 per cent. of sulphuric acid, or 0.9 per cent. of lactic acid. The acidity is increased in pregnancy and disappears entirely for six weeks after labour. When the normal thin coating of discharge on the vaginal walls either gradually or suddenly becomes yellow or greenish, mucopurulent or purulent, it is pathological and will cause, in many cases, sterility, and when the pathological discharge is cured, conception will occur. A pathological discharge allows certain organisms normally present in the vagina to flourish; they may attack the spermatozoa and destroy them.

General Malnutrition is a Common Cause of Sterility.-It means imperfect nutrition due to insufficient food or imperfect assimilation.

Gencral debilitating discases often cause temporary sterility, and when these are cured, conception will occur. I would particularly mention chronic appendicitis, septic tonsils, intestinal toxæmias.

Obesity in the Female. - It is well known that stout women do not readily conceive. The cause is probably due to some endocrinous disturbance. A large number of one-child sterilities occur in fat women owing to

- frequent abortion. In 215 cases of obesity in married women, amenorrhœa was present in 49, periods were scanty in 116 , and 2 I per cent. of these were sterile. If the obesity is treated and cured pregnancy often follows.

The next cause is what I call physiological sterility. It may happen quite naturally in the lives of some married couples, from various circumstances : coitus does not take 
place at a time corresponding to ovulation, for upon this fertility depends. Thus a man may be only able to spend one or two nights a month at his home, and these occasions may be inopportune for conception, that is inidway between the periods, for at such a time conception is very unlikely. This was proved during the late war when the short periods of leave granted to some German soldiers could be definitely correlated to the time of the menstrual cycle of their wives. It was shown that conception practically never occurs from intercourse taking place from the seventeenth to the twenty-first day after a period.

Concerning profluvium seminis, in which condition the semen is started to be expelled from the vagina following coitus. Although it is frequently associated with sterility, pregnancy very frequently occurs when it is present. Its causes are : absence of sexual orgasm in the female, a badly lacerated perineum or even a relaxed vaginal outlet. It is always present when dyspareunia is a symptom, and if the vaginal fornices are shallow. Its part as a cause of sterility is probably much over-estimated.

\section{The Investigation and Treatmen' $\mathrm{OF}$ a CASE OF STERILITy.}

In the first place, it is generally the wife who comes to her physician complaining of her sterile marriage. It is very necessary to go thoroughly into the medical history, and the points of greatest importance are :-

Age of the couple.

How long married.

Is there any history suggestive of past abdominal inflammation such as appendicitis, salpingitis, tuberculosis, peritonitis, or if a case of one-child sterility or abortion, whether there were any complications.

A past history of any of these conditions suggests amongst other things sealed tubes.

The history of any vaginal discharge is most important, whether she had the discharge beiore marriage and especially whether soon after marriage the discharge increased or altered in character, or whether frequency of micturition occurred for a short time and possibly soreness of the vulva. This suggests a venereal infection.

Question of. Intercourse.-Whether it is normal, whether any dyspareunia is present and especially whether it is followed by frequency of micturition, as this suggests an infection of Skene's tubules by the gonococcus. Whether intercourse is too frequent, if more than twice a week the spermatozoa do not have time to mature and fertilization is therefore unlikely.

The question of menstruation, whether normal, painful, irregular, or excessive.

Lastly, the Question of Preventatives.-The prolonged use of these is often followed by sterility.

If these seven points are carefully investigated, very often a good idea will be obtained as to a possible or probable cause.

The next procedure is to examine the wife.

This may result in an obvious cause being found, or on the other hand, nothing abnormal may be detected. In such a case, we must look carefully for evidence of old gonorrhœa, pus, expressible from the urethra, infection of Skene's tubules, Bartholin glands palpable, or the presence of cervicitis.

With a piece of litmus paper take the reaction of the cervical and vaginal secretions.

Let us suppose that nothing abnormal is detected. The next thing to do is to exclude the husband as the cause. This is done as follows: The wife is instructed to come again for examination within one hour of a normal coitus. It is important that intercourse should not have taken place during the previous week. A quantity of the cervical secretion is taken on a platinum loop and placed on a warm microscope slide, with a few drops of normal saline, and examined at once.

If live active spermatozoa are found, the husband is not to blame. If dead sperma- 
tozoa are found, one or two types will be seen. Spermatozoa with their tails straight out: These were alive on ejaculation and must have been killed by the cervical secretion. Or spermatozoa with their tails turned upwards: These were dead on ejaculation. To verify this obtain a condom specimen and examine under the microscope. The specimen must be kept warm or the sperm soon die.

If both parties as the result of this investigation appear to be normal, then give the following general advice, and tell them to report again in six months' time.

A separation of two months' duration. This is particularly advisable if sexual pleasure is present. If a long holiday is impossible they should sleep in separate rooms, but a holiday, especially if the wife is debilitated, is an excellent remedy.

On resumption of marital relations, advise against coitus more than twice a week. Explain that conception is most likely to occur from intercourse taking place either three days before menstruation, or during the first week afterwards.

Rahleder advises coitus during the last day of the period in some cases. Sometimes coitus in the morning rather than at night, when tired, is advisable.

If the next period does not come on to time, advise the wife to lie up until the time is either over, or the period is well started. And should the period fail to come on, further coitus must be avoided as this is one of the commonest causes of early miscarriage.

If there is a history of profluvium seminis, or the vaginal fornices are shallow, advise elevation of the buttocks after the act.

If the vaginal reaction appears to be at all hyperacid, an alkaline douche, such as bicarbonate of soda, half an ounce to the pint, should be prescribed to be used about one hour before going to bed.

Should the above fail to produce any result in the apparcntly normal, the next step is to advise a cervical dilatation together with tubal inflation, to ascertain the patency of the tubes.

While it has been known that patent tubes are essential for the occurrence of pregnancy, yet in the clinical examination attention has until recently been directed to the uterus and cervical canal alone. This neglect was due to the absence of any physical signs of any lesion of the tube on bimanual examination. Laparotomy has shown that the tubes can be sealed without any obvious deformity, either by an obstruction in its lumen or it may be blocked by fine adhesions around the ostium, and it has been shown that these adhesions of the fimbria can exist in the absence of any history of pelvic inflammation. Great advance has been made recently by the use of an apparatus which can detect the presence of an obstruction in the tubes. The method was first devised by Rubin. By means of a rather complicated apparatus, he injected gas under pressure into the uterus, if the tubes were patent it escaped into the abdominal cavity. Since he first described his apparatus, many others have been devised enabling the patency of the tubes to be tested.

If the tubes are occluded, as they will be in 50 per cent. of these cases, then laparotomy is essential to restore their patency. It is necessary to be able to answer the patient's inevitable question as to the chances of future pregnancy if she submits to a laparotomy. This depends on two things :-

(1) The Site of the Obstruction in the Tube.The nearer the ostium, or outer end of the tube this is, the better; in fact, if the obstruction is due to simply indrawing of the fimbriæ the outlook is quite good.

The Condition of the Tubal Mucous Menlbranc.-If this appears healthy, the chances are good. It is thus necessary to ascertain the site of obstruction before answering the question. This is ascertained by the transuterine injection of the tubes with lipiodol.

This; in my experience, is a much more certain and useful way of testing the patency of the tubes. In a number of cases lipiodol 
injection has proved the tubes to be patent, whereas the gas test would indicate they were sealed. Furthermore, the lipiodol injection at once gives you the site of obstruction, if present.

In addition to the diagnostic value of the inflation it has a definite curative value.

Rubin, in May, 1925, communicated to the New York State Medical Association that of $\mathrm{I}, 000$ consecutive patients insufflated alone, ninety-five had recently become pregnant. As these women reported voluntarily, it is highly probable that a systematic questionnaire would have certainly increased the numbers.

Of a total of ror first pregnancies, the result of insufflation, $2 \mathrm{I}$ had been married five to fifteen years, and $6 \mathrm{r}$ had been married one to three years. A sterile marriage of five years' duration is a fairly rigid criterion to apply.

If laparotomy is performed when the tubes are found obstructed, the condition of the ovaries should be investigated, ovarian adhesions, persistent corpora lutea, or thickened tunica albuginea, preventing ovulation, must be removed.

In conclusion, I would like to stress the point that all minor causes of sterility such as abnormal discharges and cervical erosion, must be treated as well or a satisfactory result is not to be expected. I cannot warn you too strongly that because one finds the tubes obstructed, one must immediately jump to the conclusion that this is the only cause. When we remember that the organism which attacks the tubes also attacks other areas, such as the cervix, it is obvious that these other abnormal conditions must require attention.

When this is fully realized, I am sure the results of the treatment of sterility due to infective causes will be even more satisfactory than they are to-day.

\section{POST-GRADUATE NEWS.}

Several special courses on a variety of subjects are to be held during the month of June.

From June I to June I2 a course in Gynæcology will be given at the Chelsea Hospital, occupying mornings and/or afternoons. Fee $£_{5} 5 \mathrm{~s}$.

Also from June $I$ to June 12 an afternoon course will be held at the Hospital for Diseases of the Skin, Blackfriars Road. Instruction will take place in the O.P. Department, and on both Tuesdays demonstrations of interesting cases will be given. Fee $£ \mathrm{I}$ Is.

An intensive course in medicine, surgery and the specialities will be held at the North East London Post-Graduate College (Prince of Wales's General Hospital, 'Tottenham), in conjunction with the North Middlesex Hospital, North Eastern Fever Hospital, and the L.C.C. Mental Hospital, from June I to June I3. Clinics will be held from I0.30 to 5.30 p.m. each day, including Saturday mornings. The mornings are devoted to clinical and laboratory methods, \&c., whilst the afternoons may be spent in the various departments of the Hospital. At 4.30 formal lectures will be delivered (these are open to members of the Fellowship of Medicine). This course is of especial interest to panel practitioners and to those desirous of doing revision work. Fee $£_{5} 5$ s. or $£_{3} 3$ s. for either week. Similar courses will be given on the following dates : June 22 to July 4 and July 6 to July 18 .

On successive Fridays, beginning June 5 up to and including June 26 , at 5 p.m., Dame Louise Mcllroy, D.B.E., has arranged a course consisting of four demonstrations on Ante-natal Diagnosis and Treatment at the Royal Free Hospital. Fee $f_{\mathrm{I}}$ Is.

For those interested in Tropical Diseases, an all-day comprehensive course will be 